\title{
Genetic characterisation and phylogenetic status of whipworms (Trichuris spp.) from captive non-human primates in China, determined by nuclear and mitochondrial sequencing
}

Yue Xie ${ }^{1 \dagger}$, Bo Zhao ${ }^{2 \dagger}$, Eric P. Hoberg ${ }^{3}$, Mei Li ${ }^{1}$, Xuan Zhou ${ }^{4}$, Xiaobin Gu${ }^{1}$, Weimin Lai ${ }^{1}$, Xuerong Peng ${ }^{5}$ and Guangyou Yang ${ }^{1 *}$

\begin{abstract}
Background: Whipworms (Nematoda: Trichuridae), among the most common soil-transmitted helminths (STHs), can cause the socioeconomically important disease trichuriasis in various mammalian hosts including humans and non-human primates. For many years, Trichuris from non-human primates has been assigned to the same species as the one infecting humans Trichuris trichiura. More recently, several molecular reports challenged this assumption following recognition of a Trichuris species complex observed in humans and non-human primates. A refined concept for species limits within Trichuris contributes to an understanding of diversity and the potential (zoonotic) transmission among humans and non-human primates. In this study, we expanded previous investigations by exploring the diversity of Trichuris among eight primates including three Asian autochthonous species (i.e. Rhinopithecus roxellana, Rhinopithecus bieti and Nomascus leucogenys). Species-level identification, whether novel or assignable to known lineages of Trichuris, was based on analyses of nuclear internal transcribed spacers (ITS) and mitochondrial cytochrome coxidase subunit 1 (cox1) genes.

Results: In total, seven genetically distinct subgroups of whipworms were determined to be present among the primates sampled. Most Trichuris lineages, including Subgroups 1, 1', 3, 5 and 6, showed a broad host range and were not restricted to particular primate species; in addition to T. trichiura, a complex of Trichuris species was shown infecting primates. Furthermore, it was assumed that Trichuris spp. from either N. leucogenys and $P$. hamadryas or $R$. roxellana and $R$. bieti, respectively, were conspecific. Each pair was indicated to be a discrete lineage of Trichuris, designated, respectively, as Subgroups 1 or 1' and 2, based on integrated genetic and phylogenetic evidence.

Conclusion: These results emphasise that the taxonomy and genetic variations of Trichuris are more complicated than previously acknowledged. These cumulative molecular and phylogenetic data provide a better understanding of the taxonomy, genetics and evolutionary biology of the whipworms.
\end{abstract}

Keywords: Primates, Whipworms, Trichuris, Genetic analysis, Phylogeny, Nuclear ITS, Mitochondrial cox1

\footnotetext{
* Correspondence: guangyou1963@aliyun.com

†Yue Xie and Bo Zhao contributed equally to this work.

'Department of Parasitology, College of Veterinary Medicine, Sichuan

Agricultural University, Chengdu 611130, China

Full list of author information is available at the end of the article
}

(c) The Author(s). 2018 Open Access This article is distributed under the terms of the Creative Commons Attribution 4.0 International License (http://creativecommons.org/licenses/by/4.0/), which permits unrestricted use, distribution, and

reproduction in any medium, provided you give appropriate credit to the original author(s) and the source, provide a link to the Creative Commons license, and indicate if changes were made. The Creative Commons Public Domain Dedication waiver (http://creativecommons.org/publicdomain/zero/1.0/) applies to the data made available in this article, unless otherwise stated. 


\section{Background}

Whipworms (Nematoda: Trichuridae) are among the most common soil-transmitted helminths (STHs) and cause trichuriasis in various mammalian hosts with important socioeconomic impact [1]. Humans and non-human primates, as well as mammals such as ruminants, marsupials and rodents, serve as definitive hosts [2-5]. Infection with Trichuris is based on direct transmission and generally results from oral ingestion of the infective eggs found in food, water and soil [6]. Following ingestion, the first-stage larvae (L1s) hatch and travel to the large intestine (caecum and colon) of mammalian hosts where they burrow into the intestinal mucosa and further develop, moult and propagate as dioecious adults that release eggs into the faeces. Damage to the intestinal mucosa is caused by the larval and adult worms that can lead to a variety of symptoms including typhlitis, colitis, bloody diarrhoea, growth stunting and increased susceptibility to other pathogens [7].

Currently, about 80 valid species are identified in the genus Trichuris, with most considered specific to particular taxonomic groups of hosts [8]. Among them, both species of Trichuris, namely Trichuris trichiura (Linnaeus, 1771) and Trichuris suis Schrank, 1788, are regarded as zoonotic parasites and pose threats to public health [9]. Traditional taxonomic sampling and research have focused on characterisation and differentiation of human-originated T. trichiura and pig-originated T. suis with the conclusion that these nominal taxa of whipworms are two separate but closely related species [1013]. Non-human primates, the closest human relatives, can endure high whipworm infections that are typically assumed to be $T$. trichiura according to morphological criteria [14-18]. This assumption appears reasonable because $T$. trichiura has previously been found in such primates as macaques (Macaca mulatta Zimmerman, 1780), crab-eating macaques (Macaca fascicularis Raffles, 1821) and other Old and New World monkeys [19-22].

Several recent molecular studies, however, revealed that primates including humans might host more than one species of Trichuris. For instance, Ravasi and colleagues [23] found two distinct Trichuris species occurring, respectively, in humans and baboons (Papio ursinus Kerr, 1792) based on sequencing the nuclear internal transcribed spacers (ITS, ITS1-5.8S-ITS2). Further, Callejón et al. [24] confirmed Trichuris sp. of $P$. ursinus as a new species, Trichuris ursinus Callejón, Halajian \& Cutillas, 2017, using biometrical and molecular evidence. Likewise, Hansen et al. [25] showed that humans and baboons (Papio anubis Lesson, 1827 and Papio hamadryas Linnaeus, 1758) were hosts for Trichuris species representing distinct lineages based on differentiation involving sequence analysis of a combination of ITS2 and $\beta$-tubulin genes. Furthermore, Ghai et al. [26] suggested that the whipworms infecting diurnal monkey species, chimpanzees and humans may comprise three genetically distinct Trichuris groups, with one group showing broad host range and others with a narrow spectrum of recognised hosts. Additionally, building on datasets using complete mitochondrial DNA (mtDNA), Liu et al. [27] identified a potentially novel Trichuris sp. in a non-human primate François' leaf-monkey. Another study by Hawash et al. [28], employing comparative mitogenomics, showed that multiple species of Trichuris inhabit humans and baboons. Considering that cross-infection and possible hybridisation have been documented between Trichuris spp. [12, 29], there remains considerable controversy concerning whether putative lineages in primates constitute one or more species [3]. Resolution of species limits can contribute to a stable taxonomy for species of Trichuris and therefore provides a pathway to identify the nature of host range among non-human primates and humans and the potential for zoonotic transmission.

Ongoing epidemiological surveys on potential helminthic zoonoses, centred in the southwestern zoos of China, expanded previous investigations by including specimens of Trichuris in eight species of primates including three autochthonous to Asia (i.e. Rhinopithecus roxellana Milne-Edwards, 1870; Rhinopithecus bieti Milne-Edwards, 1897; and Nomascus leucogenys Ogilby, 1840). Our goal was to characterise whipworms from an assemblage of diverse hosts relative to currently defined lineages of Trichuris facilitating (i) identification of these isolates at species level by genetically analyzing the nuclear ITS and mitochondrial cytochrome $c$ oxidase subunit 1 (cox1) markers; and (ii) determination of levels of genetic variation among these Trichuris by comparisons among those documented in other non-human primates and humans which are available in GenBank.

\section{Methods \\ Animals and parasite sampling}

A total of eight non-human primate host species, including the golden snub-nosed monkey ( $R$. roxellana), black snub-nosed monkey $(R$. bieti), vervet monkey (Chlorocebus aethiops Linnaeus, 1758), rhesus monkey ( $M$. mulatta), northern white-cheeked gibbon ( $N$. leucogenys), northern pig-tailed macaque (Macaca leonina Blyth, 1863), anubis baboon (P. anubis) and hamadryas baboon ( $P$. hamadryas) from the Chengdu Zoo (Sichuan, China) and Kunming Zoo (Yunnan, China) were sampled in this study (Fig. 1). These animals all were bred in the zoo conditions and socially raised and caged in groups, in strict accordance with good animal practices and veterinary inspection procedures. No introductions and/or translocations were permitted during the captive period. After treatment with mebendazole 20 


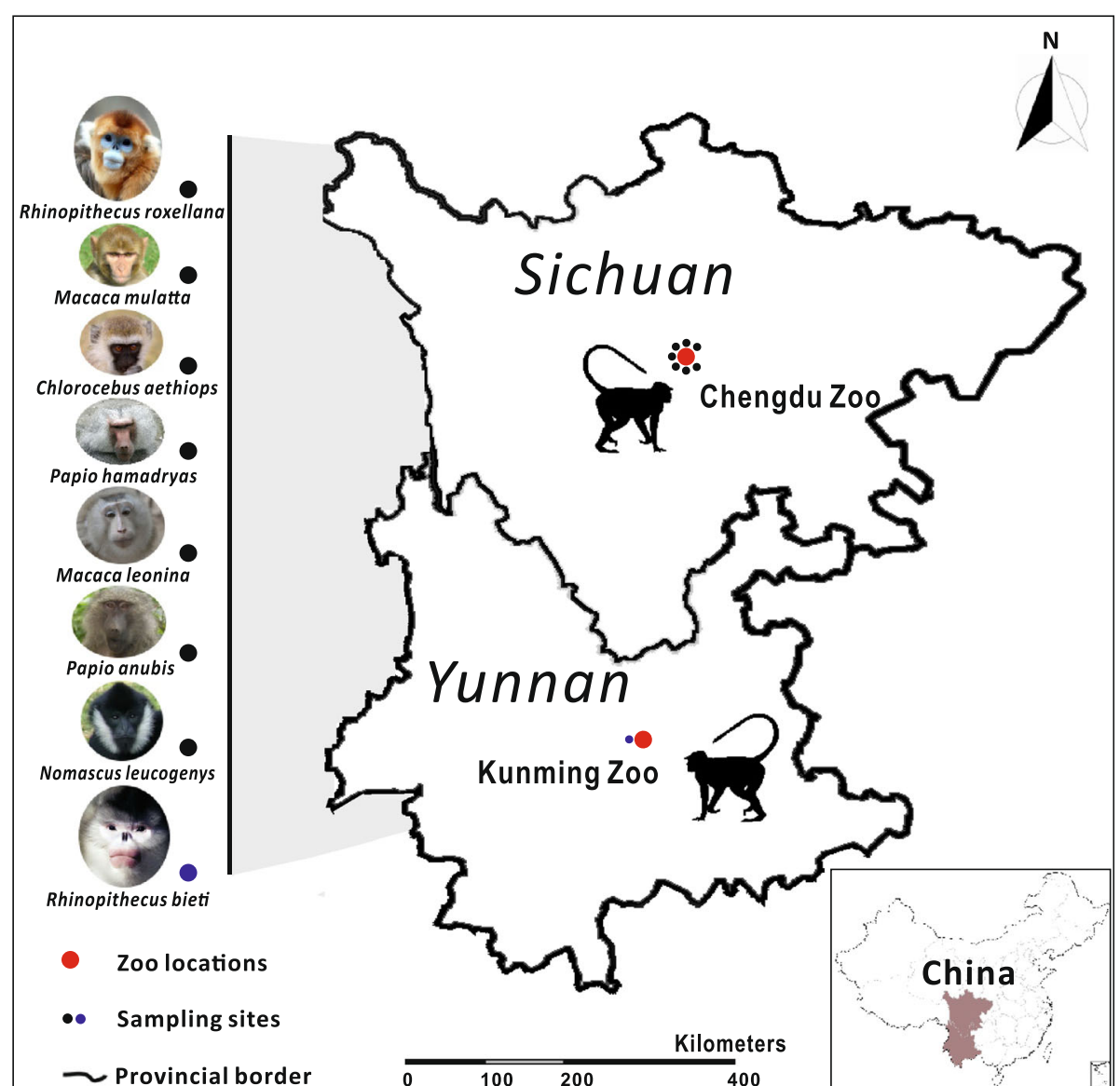

Fig. 1 Provincial map of China showing the sampling location (small black circles) of Trichuris spp. collected from eight distinct non-human primates in Chengdu Zoo in Sichuan Province and another in Kunming Zoo in Yunnan Province (large red circles)

$\mathrm{mg} / \mathrm{kg}$ daily for 3 days (B. Zhao unpublished data), the animals were temporarily separated and then 18 adult whipworm specimens were collected from naturally infected golden snub-nosed monkeys, northern white-cheeked gibbons, anubis baboons, vervet monkeys, northern pig-tailed macaques, rhesus monkeys and hamadryas baboons (Table 1). Adult worms from each host were washed separately in phosphate-buffered saline, morphologically identified to the genus level $[30,31]$, fixed in $70 \%$ (v/v) ethanol and then stored at $-20{ }^{\circ} \mathrm{C}$ until use. Whipworm eggs from faeces of two black snub-nosed monkeys were also isolated for this study because no worms were found during and after anthelmintic treatment. All non-human primate groups were sampled only once to prevent pseudo-replication of individuals. All samples were collected between January and November 2016.

\section{Sample processing and DNA isolation}

Morphology separated male and female worms. The whole male worms were used to extract genomic DNA whereas only the thin anterior part (3/5) of female worms was used for DNA isolation, as described
Table 1 Summary information of non-human primate hosts and their Trichuris parasites sampled in this study

\begin{tabular}{lll}
\hline Host species & \multicolumn{2}{l}{ Sampled numbers } \\
\cline { 2 - 3 } & Host & Parasite $^{\mathrm{a}, \mathrm{b}}$ \\
\hline $\begin{array}{l}\text { Golden snub-nosed monkey } \\
\text { (Rhinopithecus roxellana) }\end{array}$ & 1 & $1(1)$ \\
Anubis baboon (Papio anubis) & 3 & $4(1 / 1 / 2)$ \\
Vervet monkey (Chlorocebus aethiops) & 1 & $2(2)$ \\
Northern pig-tailed macaque (Macaca leonina) & 1 & $3(3)$ \\
Rhesus monkey (Macaca mulatta) & 2 & $4(2 / 2)$ \\
Northern white-cheeked gibbon & 1 & $2(2)$ \\
(Nomascus leucogenys) & & \\
Hamadryas baboon (Papio hamadryas) & 2 & $2(1 / 1)$ \\
Black snub-nosed monkey (Rhinopithecus bieti) & 2 & $2(1 / 1)$ \\
\hline
\end{tabular}

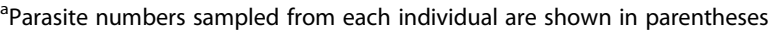
${ }^{\mathrm{b}}$ Trichuris eggs from faeces of two black snub-nosed monkeys were included in this study 
elsewhere [12, 25, 28]. For faecal eggs, a modified ethyl acetate sedimentation method was used for egg purification and concentration [32], followed by egg-shell breakage with an alternate boiling and freezing $\left(100{ }^{\circ} \mathrm{C}\right.$ and liquid nitrogen) procedure for several cycles. Worm DNA was isolated using the Universal Genomic DNA Extraction Kit Ver. 3.0 (TaKaRa Biotech, Dalian, China), and egg DNA was isolated from $200 \mu \mathrm{l}$ of sedimented faeces using the QIAamp DNA Mini Stool Kit (Qiagen, Hilden, Germany), followed by further purification with the MasterPure DNA Purification Kit (Epicentre, Madison, USA) according to the manufacturer's instructions. DNA purity and concentration were detected using a NanoDrop ND-2000 (Thermo Scientific, Wilmington, DE, USA).

\section{PCR amplification and sequencing}

The nematode parasite nuclear ITS and mitochondrial cox 1 genes were amplified to verify these Trichuris isolates using polymerase chain reaction (PCR). On the basis of the alignments of the relatively conserved regions of the congeneric species T. trichiura, $T$. suis and Trichuris muris in GenBank ${ }^{\mathrm{TM}}$ (see Table 2), the full ITS region ( 1200 bp) and a fragment ( $800 \mathrm{bp})$ of the cox1 gene were separately amplified from individual DNA samples with newly designed primers. Two PCR primer sets were as follows: ITS, forward (5'-ATC AGA ACA CAG CAA CAG-3') and reverse (5'-AAC ATC GAG GAG ACG TAC-3'); cox1, forward (5'-AAA AAT GGC TAT ATA CAG GT-3') and reverse (5'-GGG GCC AAA TAC TTT AAA T-3'). All PCR reactions were conducted in a total volume of $50 \mu \mathrm{l}$ containing $15-20 \mathrm{ng}$ of genomic DNA (gDNA), $25 \mu \mathrm{l} 2 \times$ Phusion High-Fidelity PCR Master Mix (Finnzymes, Espoo, Finland), $3 \mu \mathrm{l}$ gDNA, 3 $\mu \mathrm{l}$ of each primer $(10 \mathrm{pmol} / \mu \mathrm{l} ; \mathrm{TaKaRa})$ and $16 \mu \mathrm{l}$ of $\mathrm{ddH}_{2} \mathrm{O}$. Thermal cycling conditions carried out in a Mastercycler Gradient 5331 thermocycler (Eppendorf, Hamburg, Germany) were an initial denaturation at $94^{\circ}$ $\mathrm{C}$ for $5 \mathrm{~min}$; then for ITS, 35 cycles of $94{ }^{\circ} \mathrm{C}$ for $30 \mathrm{~s}$, $45.2{ }^{\circ} \mathrm{C}$ for $30 \mathrm{~s}$ and $72{ }^{\circ} \mathrm{C}$ for $45 \mathrm{~s}$; but for cox $1,35 \mathrm{cy}-$ cles at $94{ }^{\circ} \mathrm{C}$ for $30 \mathrm{~s}, 49{ }^{\circ} \mathrm{C}$ for $30 \mathrm{~s}$ and $72{ }^{\circ} \mathrm{C}$ for $45 \mathrm{~s}$; followed by a final step at $72{ }^{\circ} \mathrm{C}$ for $10 \mathrm{~min}$. For each amplification, samples without parasite gDNA and host DNA as negative controls were also included to test possible contamination. All PCR products were examined on agarose (1\%) gels to verify that they represented the target bands. The corrected gel-isolated amplicons were column-purified with the TIANgel Midi Purification Kit (TIANGEN, Beijing, China) and sub-cloned into Escherichia coli (DH5a) using the vector pGEMH-T (Promega, Madison, WI, USA). Positive clones were screened and sent for sequencing (Invitrogen Biotechnology Co. Ltd., Shanghai, China) using the T7 and SP6 primers. To ensure maximum accuracy, an individual clone from each specimen was sequenced three times independently. The consensus sequences were used for the following bioinformatic analyses and deposited in GenBank under the accession numbers KT344825-KT344832 and MH390359-MH390370 for ITS and KT344817-KT344824 and MH390703-MH390714 for cox1.

\section{Sequence and phylogenetic analysis}

The nucleotide sequences of ITS and cox1 among species of Trichuris in the present study were edited with BioEdit (Ibis Biosciences, Carlsbad, USA) and subjected to separate alignment with reference sequences from closely related species (Table 2), including the congeneric species $T$. trichiura, $T$. suis, $T$. muris, $T$. discolor, $T$. ovis and T. colobae as well as two outgroup species Trichinella spiralis and Trichinella britovi, using the Clustal $\mathrm{X} 1.83$ [33]. Codon-guided protein alignment manually adjusted the nucleotide alignment of $\operatorname{cox} 1$ during the procedure. Ambiguous sites/regions within these alignments were further filtered using GBlocks (http://molevol.cmima.csic.es/castresana/Gblocks_server.html) with default parameters. After Gblocks refining, both sequence alignment datasets were utilised for phylogenetic analyses using three different methods, namely neighbour-joining (NJ) (MEGA v.6.1 [34]), maximum parsimony (MP) (PAUP* 4.10b [35]) and Bayesian inference (BI) (MrBayes 3.2 [36]). NJ analysis was carried out in MEGA using either Kimura two-parameter (ITS) or Tamura-Nei $(\operatorname{cox} 1)$ as the best-to-fit substitution model. For the MP analysis, heuristic searches were executed using tree-bisection-reconnection (TBR) branch-swapping algorithm, and 1000 random-addition sequence replicates with ten trees held at each step, and finally, the optimal topology with bootstrapping frequencies (BF) was obtained using Kishino-Hasegawa [37]. In the BI analysis, the general time reversible (GTR) including gamma-distributed rate variation $(+\mathrm{G})$ and a proportion of invariable sites $(+\mathrm{I})(=$ GTR + G + I; ITS) and gamma-distributed rate variation (= GTR + G; cox 1$)$ was determined as the best nucleotide substitution models using the Bayesian Information Criteria (BIC) test in jModeltest v. 2.1.6 [38], and the trees were constructed with the Markov chain Monte Carlo $($ MCMC) method (chains $=4$ ) over 20,000,000 (ITS) or $500,000(\operatorname{cox} 1)$ generations with every 20,000 th (ITS) or 500th ( $\operatorname{cox} 1)$ tree being saved; when the average standard deviation of the split frequencies fell below $0.01,25 \%$ of the first saved trees were discarded as "burn-in" and the consensus ( $50 \%$ majority rule) trees were inferred from the remaining trees and further visualised graphically with TreeviewX [39], with nodal supports expressed as posterior probabilities (PP). T. spiralis [40] alone or in combination with $T$. britovi [41] was used as outgroup reference and included in the phylogenetic analyses. Moreover, given almost identical nucleotide sequences of both ITS and cox 1 genes observed in either worms or eggs from the 


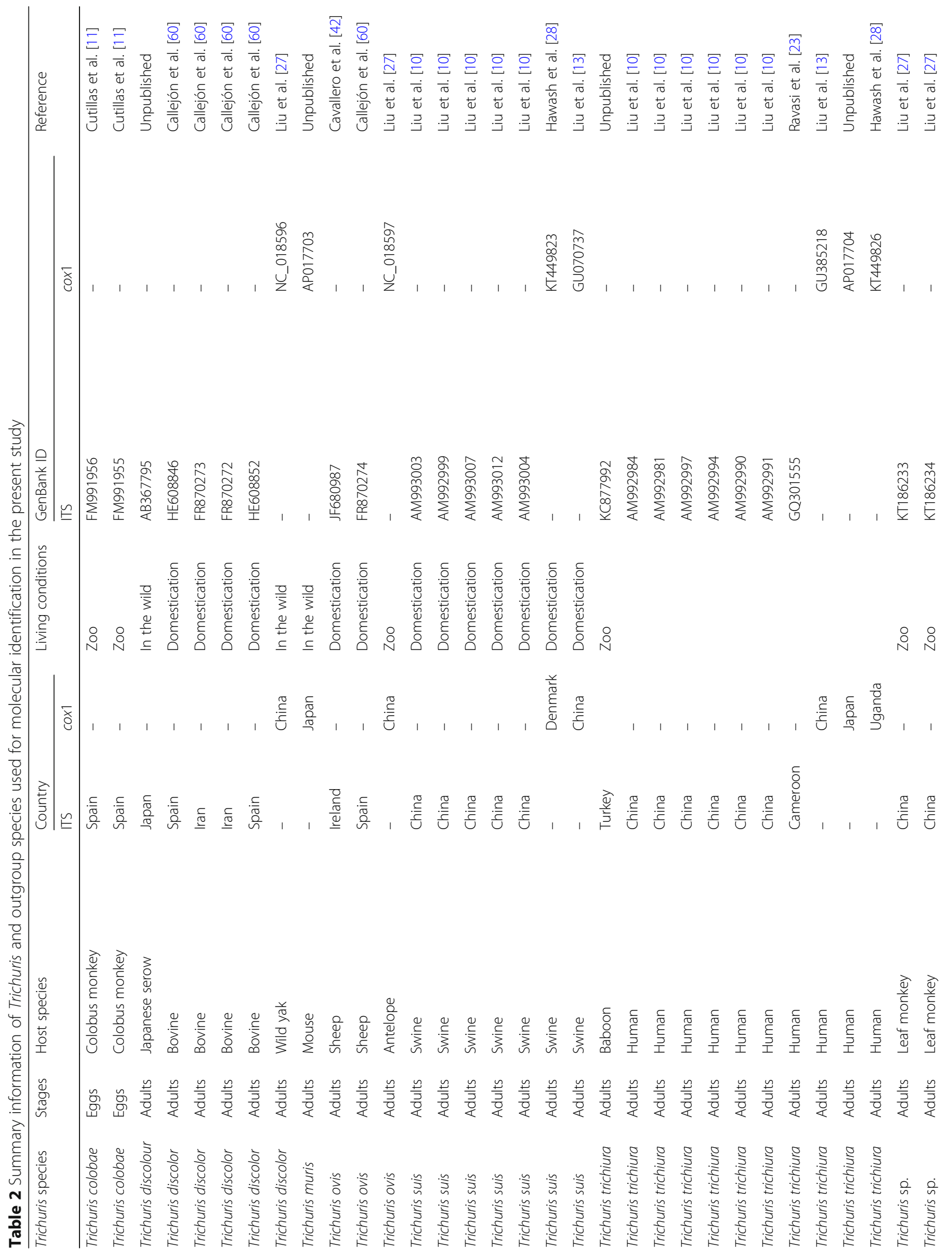




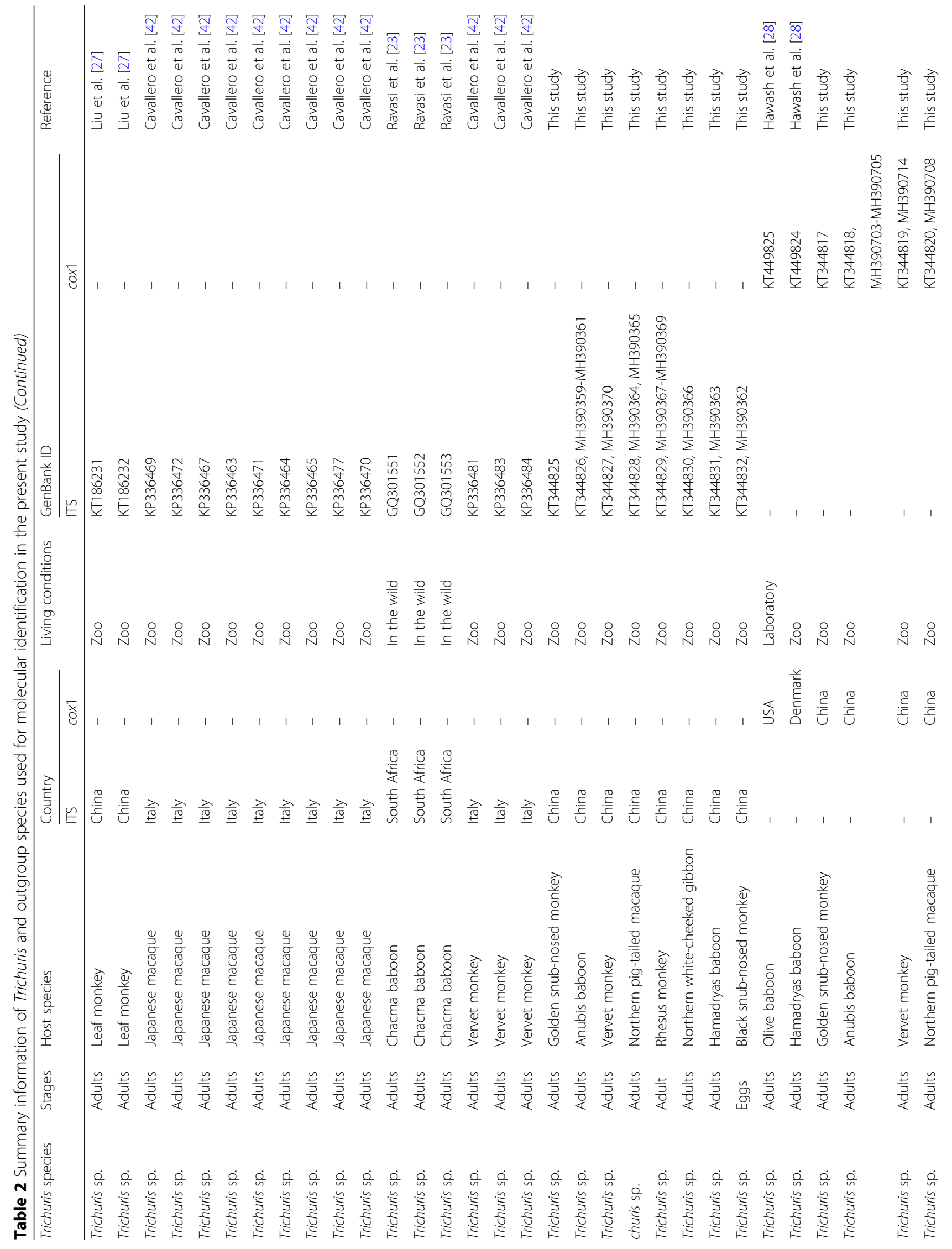




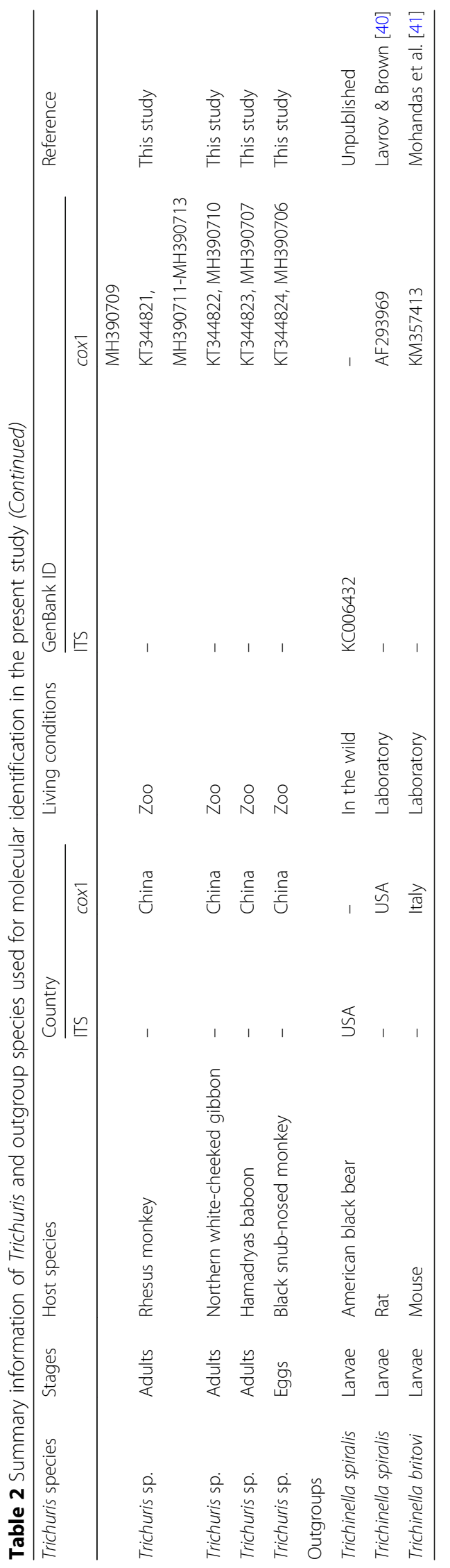


same host species in this study, one representative specimen identified here was selected and coupled with T. trichiura, T. suis, T. muris, T. discolor and T. ovis as well as two putative species of Trichuris in baboon for further detection of synonymous and non-synonymous mutations in the mitochondrial cox 1 gene based on their corresponding amino acid alignment, followed by determination of genetic distances between them using a distance matrix based on the maximum composite likelihood model in MEGA [37].

\section{Results}

\section{Sequence characterisation}

The final matrix of the ITS region obtained from each specimen of Trichuris collected from $R$. roxellana, $R$. bieti, C. aethiops, M. mulatta, N. leucogenys, M. leonina, $P$. anubis and $P$. hamadryas, respectively, was determined to be approximately $1200 \mathrm{bp}$ in length after manual assembly. The sequences of the 5 ' and 3 ' ends of ITS of these Trichuris spp. were detected by comparison with those of $T$. trichiura from human (GenBank: AM992981) and T. suis from swine (GenBank: AM992999). The full lengths of the ITS region sequenced from these twenty Trichuris isolates according to the host as mentioned above order were as follows 1205 bp, 1220 bp, 1246 bp, 1248 bp, 1202 bp, 1241 bp, $1264 \mathrm{bp}$ and $1202 \mathrm{bp}$, respectively, with a total 37.2$37.9 \% \mathrm{~A}+\mathrm{T}$ content. The nucleotide sequences of ITS in either worms or eggs from the same host species were determined to be almost identical (99.9-100\%). Sequence analysis revealed that Trichuris from N. leucogenys and $P$. hamadryas shared the highest identity (100\%), followed by $99.8 \%$ identity between Trichuris from $R$. roxellana and $R$. bieti and 95.5-97.2\% identity between Trichuris from $C$. aethiops, M. leonina and $P$. anubis. Trichuris from $M$. mulatta appeared to have the lowest sequence identity (91.8-94.6\%) compared with the other isolates. Based on the identities, there were a total of 1203 conserved sites and 105 variable sites (including 99 parsimony-informative and six singleton sites) observed in the pairwise alignment of ITS. It was noteworthy that when the congeneric human whipworm T. trichiura was added into this alignment, Trichuris from $C$. aethiops, $N$. leucogenys, $M$. leonina, P. anubis and $P$. hamadryas consistently exhibited a higher identity with $T$. trichiura than that from $R$. roxellana, $R$. bieti and M. mulatta (data not shown). For the cox1 sequences, an identical sequence length (767 bp) was found across these 20 Trichuris isolates, with an average $62.9 \% \mathrm{~A}+\mathrm{T}$ content, a typical mitochondrial nucleotide feature in nematodes (AT skewing). The base sequences of $\operatorname{cox} 1$ in either worms or eggs from the same host species were determined to be identical. Interestingly, nucleotide BLAST once again showed a high sequence identity (99.6\%) occurring between Trichuris from $N$. leucogenys and $P$. hamadryas, the same as that of Trichuris from $R$. roxellana and $R$. bieti, although a higher identity (99.9-100\%) was seen among Trichuris from the C. aethiops, M. leonina and M. mulatta (Additional file 1: Figure S1 and Additional file 2: Figure S2). Regarding identity comparisons, a total of 609 conserved sites and 158 variable sites (including 157 parsimony-informative and one singleton sites) were detected in the 767 bp pairwise alignment. Likewise, when the human-originated $T$. trichiura was introduced to the nucleotide alignment of cox1, species of Trichuris from C. aethiops, N. leucogenys, M. leonina, P. anubis and P. hamadryas and M. mulatta had higher identities (98.0-99.7\%) with T. trichiura than that of Trichuris from $R$. roxellana $(80.6 \%)$ and $R$. bieti (80.7\%). Such an identity trend was also observed in their corresponding protein comparisons (not shown).

In this context, we specifically (i) located these conserved sites in cox 1 by including other congeneric whipworms T. suis, T. muris, T. discolor and T. ovis as well as a Trichuris species in baboon Trichuris sp. and (ii) also focused on the variable sites, in order to determine if the base conservation was Trichuris lineage-specific and if there were non-synonymous substitutions apparent via comparison of their protein sequences in representative specimens. As shown in Additional file 1: Figures S1 and Additional file 2: Figure S2, we found that among 609 conserved base sites 455 were unique for the Trichuris lineage because no changes were observed across all congeneric species included in the alignment. Among 312 variable base sites, however, 204 were found unique for primate Trichuris spp. (in orange), including 66 being human T. trichiura-specific (in yellow) and 47 being non-human primate Trichuris-specific (in blue). Interestingly, among these 204 variable sites 98 were further found in two snub-nosed monkeys-specific (in red) and 24 were confirmed be non-synonymous substitutions based on protein alignments, which leaded to a total of 16 amino-acid changes, including $\mathrm{V}(\mathrm{Val}) \rightarrow \mathrm{I}$ (Ilu), M $($ Met $) \rightarrow$ L (Leu), M (Met) $\rightarrow$ I (Ilu), F (Phe) $\rightarrow$ L (Leu), $\mathrm{E}(\mathrm{Glu}) \rightarrow \mathrm{N}$ (Asn), Y (Tyr) $\rightarrow$ F (Phe), L (Leu) $\rightarrow \mathrm{I}(\mathrm{Ilu})$, $\mathrm{V}(\mathrm{Val}) \rightarrow \mathrm{M}$ (Met), M (Met) $\rightarrow \mathrm{T}$ (Thr), S (Ser) $\rightarrow \mathrm{C}$ (Cys), F (Phe) $\rightarrow$ L (Leu), S (Ser) $\rightarrow$ T (Thr), G (Gly) $\rightarrow$ $\mathrm{S}$ (Ser), S/T (Ser/Thr) $\rightarrow \mathrm{N}($ Asn) and I (Ilu) $\rightarrow \mathrm{V}$ (Val) (see Additional file 2: Figure S2).

\section{Evolutionary distance analysis}

The estimates of the evolutionary distance among these eight representative specimens of Trichuris and with other closely related whipworms were calculated and are shown in Table 3. Both datasets consistently placed species of Trichuris in $N$. leucogenys close to that in $P$. hamadryas and species of Trichuris in $R$. roxellana close to that in $R$. bieti with the minimum intraspecific evolutionary distances $(0.0000$ for ITS and 0.0030 for cox 1 
Table 3 Estimates of evolutionary distance between Trichuris spp. recovered from different host species using nuclear ITS (below diagonal) and mitochondrial cox1 (above diagonal)

\begin{tabular}{|c|c|c|c|c|c|c|c|c|c|c|c|c|c|c|c|}
\hline & Tsp_Nl & P_Ph & sp_Pa & sp_Ca & sp_Ml & Tsp_Mm & Tsp_Rr & Tsp_Rb & Tsp & Ttr & Tsu & Tmu & Tdi & Tov & \\
\hline sp_NI & & 0.0030 & .0144 & 0.0175 & 0.0165 & 0.0165 & 0.1882 & 0.1895 & 0.1590 & 0.1423 & 0.2370 & 0.2275 & 0.2636 & 0.2771 & Tsp_NI \\
\hline 11 & 0.0000 & & 0.0154 & 6 & 0.0176 & 0.0176 & 8 & 0.1911 & 0.1575 & 9 & 0 & 5 & 6 & 11 & h \\
\hline P_Pa & 0.0056 & 0.0056 & & 0 & 0.0040 & 0.0040 & 1911 & 1924 & 545 & 1352 & 0.2338 & 0.2243 & 2536 & 0.2740 & sp_Pa \\
\hline P- & 070 & 078 & 0067 & & 0 & 0.0010 & 6 & 0.2000 & 0.1586 & 55 & 37 & 8 & 59 & 55 & $\mathrm{Ca}$ \\
\hline P_Ml & 0.0180 & 0.0180 & 0.0191 & 0.0191 & & 0.0000 & 0.1972 & 0.1985 & 0.1572 & 0.1352 & 0.2372 & 0.2243 & 2553 & 0.2740 & D_Ml \\
\hline ר & 0.0340 & 0.0340 & 0.0352 & 29 & 9 & & 0.1972 & 0.1985 & 0.1572 & 0.1352 & 2 & 3 & 3 & 0 & Tsp_M \\
\hline P_Rr & 0.0191 & 0.0191 & 0.0120 & 0.0120 & 0.0179 & 0.0340 & & 0.0030 & 0.2205 & 0.2004 & 0.2439 & 0.2365 & 0.2702 & 0.2421 & Tsp_Rr \\
\hline Tsp_Rb & 0 & 1 & 2 & 4 & 0 & 0.0283 & 22 & & 2174 & 0.1988 & 07 & 54 & 5 & 9 & $\operatorname{Tsn} B$ \\
\hline p & 0.0067 & 0067 & 0.0033 & 0.0033 & 0.0180 & 0.0317 & 0.0089 & 0.0214 & & 39 & 0.2515 & 0.2435 & 0.2782 & 0.2808 & $\mathrm{~T}$ \\
\hline Trr & 0.0044 & 0.0044 & 0.0033 & 0.0056 & 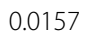 & 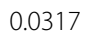 & 0.0044 & 0.0168 & 44 & & & 5 & 3 & 4 & Ttr \\
\hline Tsu & 0.4043 & 0.4043 & 0.4019 & 0.3966 & 0.3883 & 0.3851 & 0.4004 & 0.3948 & 0.3983 & 0.3963 & & 0.0635 & 0.2418 & 0.2546 & Tsu \\
\hline 等 & 0 & 0.4228 & 0.4203 & 0.4240 & 0.4178 & 0.4086 & 0.4188 & 0.4191 & 0.4185 & 2 & 46 & & .2422 & 2 & Tmu \\
\hline di & 0.6228 & 0.6228 & 0.6286 & 0.6217 & 0.6126 & 0.6098 & 0.6 & 194 & 0.6217 & 0.6211 & 0.5624 & 0.5699 & & 0.1737 & Tdi \\
\hline \multirow[t]{2}{*}{ Tov } & 32 & 0.6282 & 0.6342 & 0.6272 & 0.6179 & 0.6175 & 0.6230 & 0.6236 & 72 & 56 & 55 & 35 & 0.0622 & & v \\
\hline & Tsp_NI & Tsp_Ph & Tsp_Pa & Tsp_Ca & Tsp_Ml & Tsp_Mm & Tsp_Rr & Tsp_Rb & Tsp & Ttr & Tsu & Tco & Tdi & Tov & \\
\hline
\end{tabular}

Note: Evolutionary distances between Trichuris spp. from eight non-human primates included in this study are highlighted in boldface for ITS- and cox1-based estimates, respectively. Given almost identical nucleotide sequences of both ITS and cox 1 genes in either worms or eggs from the same host species, eight representative specimens were used to calculate evolutionary distances using a maximum composite likelihood model

Abbreviations: Tsp_Nl, Trichuris sp. from Nomascus leucogenys; Tsp_Ph, Trichuris sp. from Papio hamadryas; Tsp_Pa, Trichuris sp. from Papio anubis; Tsp_Ca, Trichuris sp. from Chlorocebus aethiops; Tsp_Ml, Trichuris sp. from Macaca leonina; Tsp_Mm, Trichuris sp. from M. mulatta; Tsp_Rr, Trichuris sp. from Rhinopithecus roxellana; Tsp_Rb, Trichuris sp. from Rhinopithecus bieti; Tsp, Trichuris sp.; Ttr, Trichuris trichiura; Tsu, Trichuris suum; Tco, Trichuris colobae; Tmu, Trichuris muris; Tdi, Trichuris discolor; Tov, Trichuris ovis

between the former; 0.0022 for ITS and 0.0030 for cox 1 between the latter), in accordance with conclusions of our identity analysis. Among four other Trichuris isolates that were in $C$. aethiops, $M$. leonina, $P$. anubis and M. mulatta, respectively; however, our analysis indicated that their evolutionary distances varied by different genetic makers used. For example, Trichuris spp. in M. leonina and $M$. mulatta showed a maximum intraspecific evolutionary distance (0.0329) in ITS-based analysis while the value changed into 0.0000 in the cox 1 data (see Table 3). Distances estimated between these eight representative specimens of Trichuris and other related congeners indicated the highest similarity to human $T$. trichiura and baboon Trichuris sp. with 0.0033-0.0317 (ITS) and 0.1352-0.2205 (cox1); more profound divergence characterised comparisons to T. suis with 0.38510.4043 (ITS) and 0.2338-0.2407 (cox1), T. discolor with $0.6098-0.6286$ (ITS) and 0.2553-0.2705 (cox1) and T. ovis with $0.6175-0.6282$ (ITS) and 0.2389-0.2755 (cox1) (Table 3).

\section{Phylogenetic characterisation}

The interrelationships of these 20 specimens of Trichuris and their phylogenetic relationships with other related species were inferred from the respective sequences of ITS and cox 1 using NJ, MP and BI algorithms and their corresponding tree topologies are depicted in Figs. 2, 3.
Although the two identical trees (NJ/MP/BI) topologically differed from each other because of genes and reference species included here, both analyses provided a consistent, robust phylogenetic resolution for these 20 isolates and their congeneric species in the genus Trichuris. Unequivocal subclades representing seven groups of Trichuris were demonstrated, showing varying patterns of broad to relatively narrow host range. Specifically, (i) Trichuris specimens in $N$. leucogenys and $P$. hamadryas clustered together and formed a sister cluster with other Trichuris in C. aethiops, $P$. anubis and $M$. leonina as well as other primates including humans, with robust support for both tree topologies (all bootstrap values $\geq 83$ or 0.88 ); we named such clades as Subgroups 1 (Fig. 2) or 1' (Fig. 3). (ii) Trichuris isolates in $R$. roxellana and $R$. bieti individually grouped as another clade with high statistical support (all values $\geq 96$ or $=1.00$ ) and were referred as Subgroup 2 in both ITS and cox1. (iii) Trichuris sp. in M. mulatta grouped with whipworms in the Japanese macaque Macaca fuscata Blyth, 1875 as a separate clade designated as Subgroup 3 in the ITS-based analysis but clustered with whipworms in $C$. aethiops, $M$. leonina, $P$. anubis, $P$. hamadryas, $N$. leucogenys and humans as an independent clade (Subgroup $\left.1^{\prime}\right)$ in the $\operatorname{cox} 1$ data, with a very similar host range to Subgroup 1 in the ITS. (iv) Four isolates of Trichuris in the François' leaf monkey Trachypithecus françoisi 


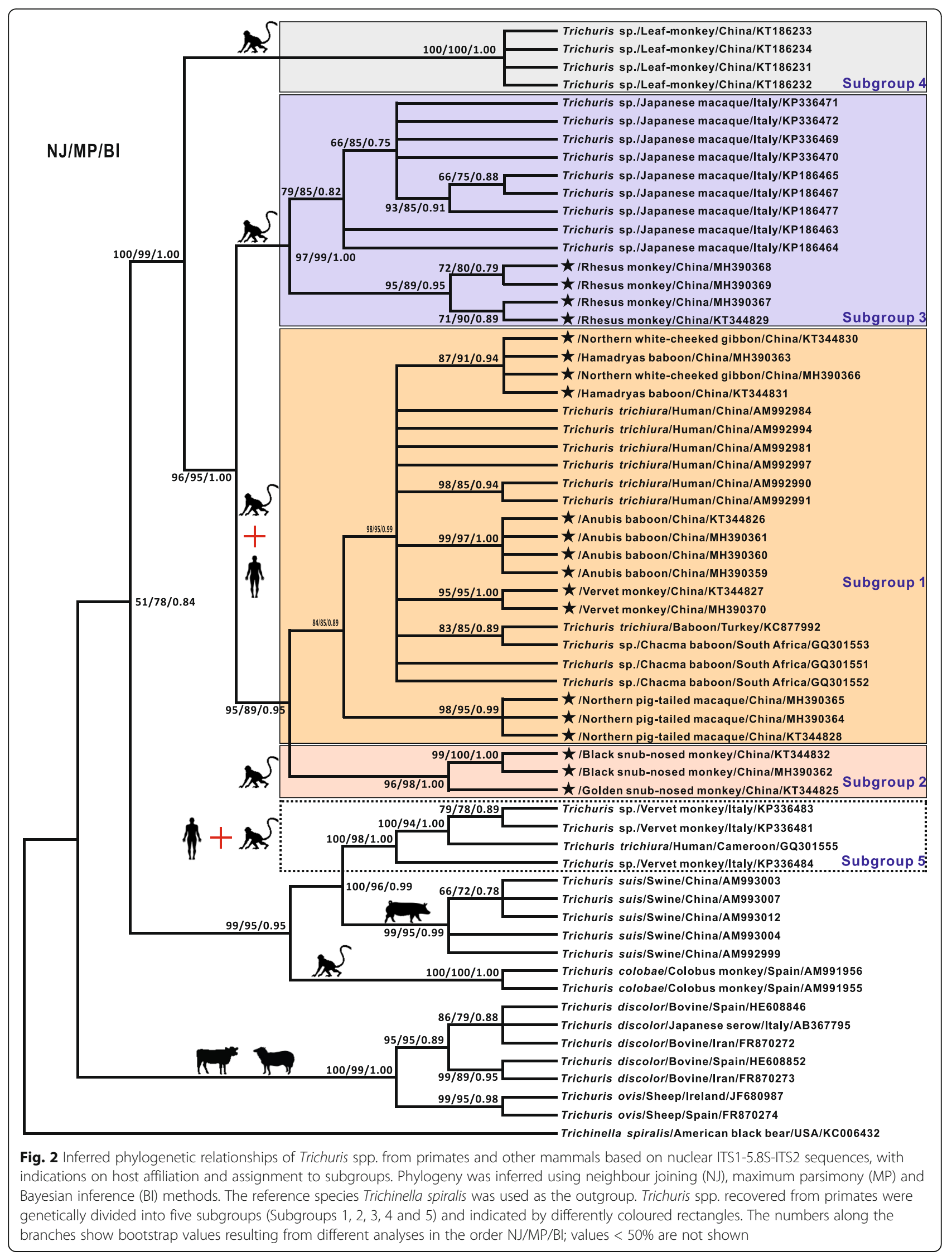




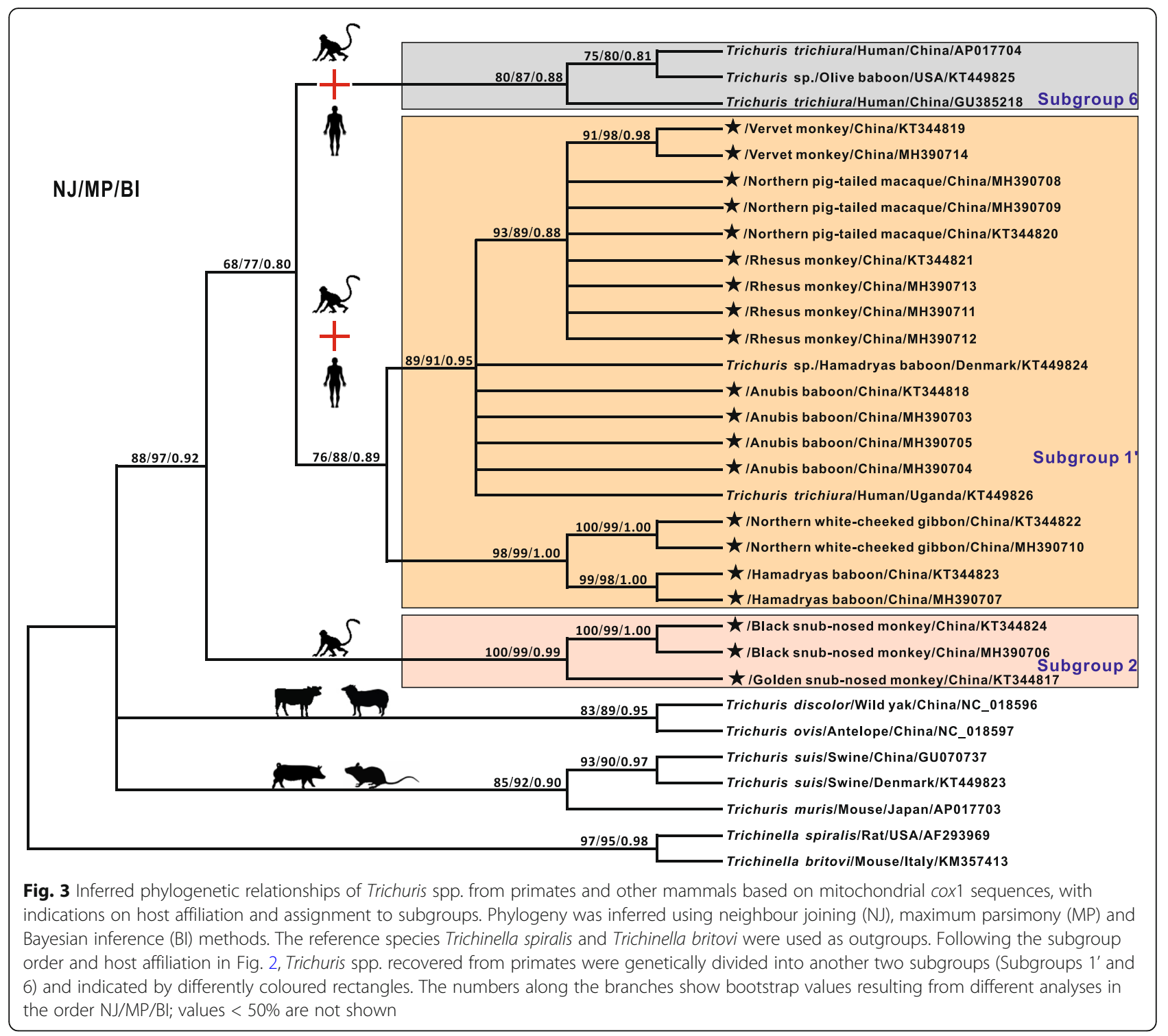

(Pousargues, 1898) clustered together and formed a monophyletic group with all other primate whipworms included in this study and were referred as Subgroup 4 based on ITS data (Fig. 2). (v) Furthermore, one human-derived Trichuris grouped with the whipworms in vervet monkeys and together were closely related to T. suis and were referred to as Subgroup 5 in the ITS (Fig. 2), while two human-derived Trichuris grouped with the whipworm in a laboratory olive baboon and were named as Subgroup 6 in the $\operatorname{cox} 1$ (Fig. 3). (vi) For the inter-relationships of Trichuris spp. from these primates with $T$. suis from swine, $T$. muris from mice, $T$. discolor from bovines and T. ovis from sheep, excluding Subgroup 5, their phylogenetic topologies were consistent with previously proposed molecular phylogenies of the whipworms based on the nuclear and mitochondrial DNA data $[13,26,28,42,43]$, demonstrating the phylogenetic stability of these paraphyletic subgroups characterised in the present study.

\section{Discussion}

Soil-transmitted Trichuris are among the most common parasitic nematodes found in mammals, including humans and non-human primates, and the etiological agent is usually considered as T. trichiura. Recent molecular epidemiological investigations, however, reveal that $T$. trichiura is not the only species of Trichuris to infect humans and non-human primates, suggesting the existence of a Trichuris species complex in primates. Given previously limited primate sampling and possible cross-infection and/or hybridisation among species of Trichuris [12, 29], the question about Trichuris as a large complex of distinct species rather than as a limited number of congeners in primates remains unclear. In 
the present study, the whipworms from an additional eight non-human primate hosts were included and combined with current existing data for Trichuris lineages in humans and other non-human primates.

Twenty whipworm isolates in this study were identified as Trichuris according to morphological characters $[30,31]$. However, given current limited morphological diagnostic characters among Trichuris spp. [42] and a common phenomenon of morphological convergence in intestinal nematodes (e.g. Ascaris spp. [42, 44, 45] and Anisakis spp. [46]), the application of molecular markers for species identification was emphasised. For instance, the ITS of the nuclear ribosomal DNA is considered as a useful genetic marker for resolving nematode relationships at the species level [47]. Through pairwise comparisons of ITS among these twenty Trichuris isolates, we found that Trichuris spp. in N. leucogenys and P. hamadryas had an identical nucleotide sequence, suggesting that both hosts may harbour the same Trichuris species. A similar conjecture was also made for another pair of Trichuris spp. in $R$. roxellana and $R$. bieti due to the extremely high nucleotide identity (99.8\%). To confirm these findings, ITS-based phylogenetic analyses (NJ/MP/ $\mathrm{BI})$ were performed and the results once again showed that species of Trichuris from the two pairs of hosts clustered together separately, with high bootstrap values (see Fig. 2), supporting the contention that $N$. leucogenys and $P$. hamadryas or $R$. roxellana and $R$. bieti, respectively, have their own Trichuris spp. Encouragingly, the same conclusions were further strengthened by analysis of another genetic marker, i.e. the mitochondrial cox1. It should be noted that cox 1 analysis was added because numerous studies of nucleotide substitution in nematode mitochondrial genes (e.g. $\operatorname{cox} 1$ and nad4) have proven that they have utility in identifying and discovering novel or cryptic species among very closely related taxa due to their faster mutation rates relative to nuclear genes, maternal inheritance and lack of recombination [48-50]. Similar to the nuclear ITS, a comparable nucleotide identity (99.6\%) was observed in cox1 of Trichuris spp. in either $N$. leucogenys and $P$. hamadryas or $R$. roxellana and $R$. bieti; more importantly, high bootstrap support was evident, based on phylogenetic analyses (NJ/MP/BI) of $\operatorname{cox} 1$ that demonstrated the same species of Trichuris in either $N$. leucogenys and $P$. hamadryas or $R$. roxellana and $R$. bieti (Additional file 2: Figure S2, Fig. 3). Nevertheless, possible cross-infection could be a confounder for this outcome because of the close or sympatric housing conditions in the zoos. This possibility was ruled out because sampling records, particularly for these two snub-nosed monkeys showed that $R$. roxellana was housed in the Chengdu Zoo (Sichuan, China) whereas $R$. bieti was kept in Kunming Zoo (Yunnan, China). Additionally, the captive history evidenced that these host populations were bred in the zoos and no translocations and/or introductions were reported before, suggesting that these Trichuris spp. were present with the original captive populations of these non-human primates and their infection cycles may have been maintained over time.

Based on the integrated molecular evidence, we propose that Trichuris spp. from either $N$. leucogenys and $P$. hamadryas or $R$. roxellana and $R$. bieti may represent the same species but belong to different genetic groups in the genus Trichuris, i.e. Trichuris sp. of N. leucogenys and P. hamadryas is within Subgroups 1 and 1' and Trichuris sp. of $R$. roxellana and $R$. bieti is within Subgroup 2 (see Figs. 2, 3). Conversely, previous morphological studies showed that there were two different types of whipworms in $R$. roxellana and $R$. bieti. Specifically, Trichuris rhinopiptheroxella (Zhu et al., 2000) occurred in $R$. roxellana (also known Sichuan snub-nosed monkey) [51], and the congeneric species Trichuris rhinopithecus (Hou \& Peng, 1989) occurred in R. bieti (also known Yunnan snub-nosed monkey) [52]. Both species of Trichuris were considered valid in terms of different lengths of vasa efferentia, ejaculatory duct, cloaca and spicules and locations of the ovary [51]. It seems remarkable that $T$. rhinopiptheroxella and $T$. rhinopithecus are heterogenic based on these morphological differences. However, current increased epigenetics-based evidence about phenotypic plasticity of parasites appears to be challenging the traditional morphological taxonomy [53]. Phenotypic plasticity is the concept that one genome can produce different phenotypes, usually in response to altered environmental conditions [54]. In fact, like vertebrates, invertebrate parasites also possess the ability to modify some aspects of their morphology and physiology adapting to different conditions including the external environment and inside environment of the host [55-57]. In such cases, phenotypic plasticity, based on morphometric criteria, has been purportedly expressed in many parasites. What is even more noteworthy is that phenotypic plasticity is particularly apparent among parasitic helminths because their range of hosts, origins and developmental stages can facilitate very different morphological and physiological characters useful for adaption and survival [58]. For example, within worms, Mati and colleagues [59] found that adult males of the human parasite Schistosoma mansoni maintained in AKR/J and Swiss mice, respectively, had significant morphometric changes in the total body length and reproductive characteristics (including the extension of testicular mass and number of testes) and that these morphometric alterations were also observed when compared to S. mansoni obtained from hamsters (Mesocricetus auratus), the host in which the parasite had been adapted and maintained for decades. 
Nevertheless, all of these different phenotypes are products of the same genome including nuclear and mitochondrial DNAs. Thus, if taking the potential phenotypic plasticity of Trichuris spp. into account and combining with the aforementioned genetic evidence, our proposal that the same Trichuris species occurs in either $N$. leucogenys and $P$. hamadryas or $R$. roxellana and $R$. bieti is reasonable. Of course, additional information regarding ultrastructure and genomics of these Trichuris species and other related whipworms as well as broader taxonomic comparisons is still required to provide an increasingly precise morphological and molecular basis for species identification among whipworms. Additionally, there were 16 species-specific non-synonymous base substitutions detected in cox1 genes of Trichuris species in both $R$. roxellana and $R$. bieti (Additional file 2: Figure S2) that were further confirmed to be fixed after homologous comparisons with other congeneric species.

Phylogenetic analysis divided all primate Trichuris spp., including these 20 whipworms in this study, into seven distinct subgroups (Figs. 2, 3). Within ITS-based Subgroup 1, most whipworms were obtained from non-human primates (white-cheeked gibbons, baboons and monkeys) and clustered closely with either human-originated or baboon-derived T. trichiura. For instance, the whipworms in vervet monkey and anubis baboons shared a close relationship with human T. trichiura (Chinese isolates) than that of other non-human primates. Likewise, three specimens of Trichuris in chacma baboons from South Africa were also found to be more close to one baboon-derived $T$. trichiura (Turkey isolates) than others. Combined, these Trichuris spp. in chacma baboons from South Africa along with the whipworms in white-cheeked gibbon, hamadryas baboon, anubis baboon, vervet monkey and pig-tailed macaque from China were all grouped as part of the phylogenetically close $T$. trichiura clade. Such a conclusion would expand the host range of the 'Clade DG' as proposed by Ravasi et al. [23] and suggests that not only T. trichiura but multiple Trichuris species infect these host species. Referring to ITS-based Subgroup 1, since similar primate host cluster (excluding rhesus monkeys) was also found in cox1-based phylogeny we designated the Trichuris lineage as Subgroup 1'. In contrast to Subgroup 1, this subgroup placed Trichuris sp. in anubis baboons from China and Trichuris sp. in hamadryas baboon from Denmark more closely with human T. trichiura (Ugandan isolates) than Trichuris spp. in white-cheeked gibbon, hamadryas baboon, rhesus monkey, pig-tailed macaque and vervet monkey from China. This result is in agreement with a recently published study using partial (372 bp) cox 1 sequences by Hawash et al. [28] and further supports that whipworms in primates comprise a Trichuris species complex. Under such hypothesis, compared with Subgroup 6, it seems that Subgroup 1' reflects another fact that the human Trichuris from Uganda was genetically very distinct from that from China because both geographical isolates were found in two separate clades (see Fig. 3), suggesting at least two Trichuris spp. infecting humans. Besides, it is also noteworthy that among Subgroup 1' Trichuris sp. in rhesus monkey clustered with that of pig-tailed macaque and vervet monkey while the rhesus monkey isolates grouped with whipworms in the Japanese macaque in ITS-based Subgroup 3. This discordance in the nuclear and $\mathrm{mt}$ phylogenies is surprising and may be owing to a higher rate of nucleotide change in mt DNA of nematodes than that seen in nuclear-encoded sequences, as reported in other Trichuris spp. [60], Caenorhabditis spp. [61] and Baylisascaris spp. [62].

Also, Subgroup 2 comprising two whipworms in $R$. roxellana and $R$. bieti, Subgroup 4 containing four specimens of Trichuris sp. in François' leaf monkeys and Subgroup 5 having three vervet monkey-derived Trichuris and one human-derived Trichuris, three clades appear to be three independent lineages. These clades are genetically distant from other Trichuris species in white-cheeked gibbon, baboons and pig-tailed macaque, suggesting different species of Trichuris exist among non-human primates, as recently reported by Hawash et al. [28] and Liu et al. [27]. Previous studies have shown that there may be several Trichuris species parasitising primates, with some species only infecting non-human primates and others infecting both non-human primates and humans. Variation in host range reflects the interaction of trends in generalisation and specialisation (oscillation), the opportunity for host colonisation and capacity to establish infection as reflected in outcomes for ecological fitting in sloppy fitness space $[63,64]$ and more broadly the recently proposed Stockholm Paradigm (e.g. $[65,66])$. Faunal mixing and the potential for exchange are facilitated in zoo-park situations that may bring phylogenetically disparate assemblages of hosts and their parasites into proximity. For parasites with a direct life-cycle such as Trichuris, this establishes the potential for considerable opportunities for host colonisation that may not be apparent in natural settings. A breakdown of mechanisms for ecological isolation as demonstrated in zoo-park environments emphasise the potential for extensive colonisation processes that may result and the possibility of new disease through the exchange of parasites over time. Moreover, ITS- or cox1-based phylogenies consistently showed that $T$. trichiuraincluded clades include Subgroups 1, 1, 5 and 6. This finding should enhance public alertness to whipworms in these hosts and raise concerns about animal and human health. 
As a part of epidemiological surveys of captive non-human primates and species diversity among associated Trichuris whipworms in Chinese zoos, a number of questions are apparent: (i) what are the potential routes of parasite introduction and establishment (natural or cross infections); (ii) what is the most appropriate pathway for surveillance of parasite infections, particularly when different cryptic species of helminths may be involved; (iii) what are the most appropriate strategies to prevent parasite transport in the space-limited and to an extent artificial zoos; (iv) what are the limits and facilitators for zoonotic risk. Given that the recognised host range (defined by opportunity) of a parasite is almost always a reduced subset of the actual host range (defined by capacity to use host-based resources) that is possible, the current study provides some quasi-experimental insights about the role of breakdown in isolation and ecological fitting in expansions of host range. These are characteristics most often associated with patterns of emerging infectious diseases in the context of ecological disruption, suggesting direct lessons about the potential versus realised current host range for many species of Trichuris and the risk of zoonotic infections. Historically within the assemblage of Trichuris which circulate in humans and non-human primates, bouts of host colonisation and oscillations in host range, appear as prominent contributors to faunal assembly and diversity $[66,67]$. Therefore, there is an urgent need to clarify the Trichuris species which infect primates in order to discover transmission routes and establish suitable control measures.

\section{Conclusions}

In this study, based on analyses of the nuclear ITS and mitochondrial cox 1 datasets, we suggest at least seven genetically distinct subgroups of Trichuris present among primates including humans, supporting a previous proposal that a complex of Trichuris species other than T. trichiura infects these hosts. Furthermore, combined evidence of genetic distance analysis and phylogenies revealed that an identical whipworm species might exist either between the white-cheeked gibbon and hamadryas baboon or Sichuan and Yunnan snub-nosed monkeys. However, such a proposal requires further examination based on broad sampling and extensive morphological and genetic comparisons. Taken together, the results presented in this study once again emphasise that the taxonomy and genetics of Trichuris spp. in primates are complex and these cumulative molecular and phylogenetic data should contribute to a better understanding of the taxonomy, genetics and evolutionary biology of the whipworms.

\section{Additional files}

\begin{abstract}
Additional file 1: Figure S1. Alignment of the nucleotide sequence of mitochondrial cox 1 genes of eight representative isolates of Trichuris identified in this study and congeneric species. Nucleotide sequences of cox1 genes were retrieved from the GenBank database and aligned using the Clustal X 1.83 software. Species abbreviations and accession numbers (in parentheses) are indicated as follows: Tsp_Ph (DK), Trichuris sp. from Papio hamadryas (KT449824); Tsp_Ph (CN), Trichuris sp. from Papio hamadryas (KT344823); Tsp_NI Trichuris sp. from Nomascus leucogenys (KT344822); Tsp_Pa, Trichuris sp. from Papio anubis (KT344818); Tsp_Ml, Trichuris sp. from Macaca leonie (KT344820); Tsp_Mm, Trichuris sp. from M. mulatta (KT344821); Tsp_Ca Trichuris sp. from Chlorocebus aethiops (KT344819); Tsp_Rr Trichuris sp. from Rhinopithecus roxellana (KT344817) and Tsp_Rb Trichuris sp. from Rhinopithecus bieti (KT344824); Ttr, Trichuris trichiura (GU385218); Tsu, Trichuris suum (GU070737); Tmu, Trichuris muris (AP017703); Tdi, Trichuris discolor (NC_018596); Tov, Trichuris ovis (NC_018597). The two abbreviations DK and CN denote different geographical origins, Denmark and China, respectively. Pairwise comparisons between Trichuris spp. identified in this study were highlighted in grey. (PDF $381 \mathrm{~kb}$ )
\end{abstract}

Additional file 2: Figure S2. A simultaneous alignment of nucleotide and amino acid sequences of mitochondrial cox 1 genes of eight representative isolates of Trichuris identified in this study and their congeneric species. Building on alignments from Figure S1, the corresponding amino acid sequences inferred according to the Invertebrate Mitochondrial Code were added and aligned. Regions of identity in either nucleotide (.) or amino-acid (:) are indicated. The variable base loci unique for primate Trichuris spp. are highlighted in orange; among those is human T. trichiura-specific that are highlighted in yellow and non-human primate Trichuris-specific highlighted in blue. Further, 98 variable base loci unique for two snub-nosed monkeys are identified (in red) in order to test the non-synonymous substitutions, and a total of 16 amino-acid changes: V (Val)/I (llu), M (Met)/L (Leu), M (Met)/I (llu), F (Phe)/ $\mathrm{L}(\mathrm{Leu}), \mathrm{E}(\mathrm{Glu}) / \mathrm{N}(\mathrm{Asn}), \mathrm{Y}(\mathrm{Tyr}) / \mathrm{F}$ (Phe), L (Leu)/I (llu), V (Val)/M (Met), M (Met)/T (Thr), S (Ser)/C (Cys), F (Phe)/L (Leu), S (Ser)/T (Thr), G (Gly)/S (Ser), $\mathrm{S} / \mathrm{T}(\mathrm{Ser} / \mathrm{Thr}) / \mathrm{N}$ (Asn) and I (llu) N (Val) are observed and targeted with a black star. Percentages of nucleotide identities between these eight representative isolates of Trichuris are shown at the end of each sequence, with a number tag: (1) Trichuris sp._Ph, (2) Trichuris sp._Pa, (3) Trichuris sp. NI, (4) Trichuris sp. MI, (5) Trichuris sp. Mm, (6) Trichuris sp._Ca, (7) Trichuris sp._Rr, (8) Trichuris sp._Rb. (PDF 420 kb)

\section{Abbreviations}

BI: Bayesian inference; BIC: Bayesian Information Criteria; cox1: Cytochrome $c$ oxidase subunit 1; ITS: Internal transcribed spacers; MCMC: Markov chain Monte Carlo; MP: Maximum parsimony; mtDNA: Mitochondrial DNA; NJ: Neighbor-joining; PCR: Polymerase chain reaction; PP: Posterior probabilities; STHs: Soil-transmitted helminths; TBR: Tree-bisectionreconnection

\section{Acknowledgements}

We thank Dr Joseph F. Urban, Jr., from USDA-ARS, Beltsville Human Nutrition Research Center, Diet, Genomics and Immunology Laboratory for manuscript review and advice.

\section{Funding}

This work was supported by grants from the Programme for Changjiang Scholars and Innovative Research Team in University (PCSIRT) (Grant no. IRT0848), the Research Fund for the Chengdu Research of Giant Panda Breeding (Project no. CPF2012-13) and the High-level Scientific Research Foundation for the Introduction of Talents of Sichuan Agricultural University (no. 03120322). The funders had no role in the design, decision to publish, or preparation of the manuscript.

\section{Availability of data and materials}

The data supporting the conclusions of this article are included within the article and its additional files. Nucleotide sequences reported in this article are available in the GenBank database under the accession numbers 
KT344825-KT344832 and MH390359-MH390370 for ITS and KT344817KT344824 and MH390703-MH390714 for cox1.

\section{Authors' contributions}

YX and GYY conceived and designed the experiments. YX, BZ and ML performed the experiments, including $P C R$, cloning and sequencing. $Y X, X Z$, $X B G$ and $M L$ carried out the data analysis. BZ, WML and PXR contributed reagents/materials/analysis tools. YX, EPH and GYY wrote the initial manuscript. All authors read and approved the final manuscript.

\section{Ethics approval and consent to participate}

This study was approved by the Animal Ethics Committee of Sichuan Agricultural University (Ya'an, China; approval no. 2011-028). All parasite samples were collected with the permission of the zoos, and all procedures were performed in compliance with the "Guidelines and Recommendations for the Care and Use of Laboratory Animals of the Ministry of Health of the People's Republic of China" and "Wild Animal Protection Law of the People's Republic of China" (released in 1989). Additional details of animal welfare and steps taken to ameliorate suffering were in accordance with the recommendations of the Weatherall report, "The use of non-human primates in research". During the study, no animal died or was sacrificed.

\section{Consent for publication}

Not applicable.

\section{Competing interests}

The authors declare that they have no competing interests.

\section{Publisher's Note}

Springer Nature remains neutral with regard to jurisdictional claims in published maps and institutional affiliations.

\section{Author details}

${ }^{1}$ Department of Parasitology, College of Veterinary Medicine, Sichuan Agricultural University, Chengdu 611130, China. ${ }^{2}$ Chengdu Zoo, Chengdu 610081, Sichuan, China. ${ }^{3}$ Division of Parasitology, Museum of Southwestern Biology, University of New Mexico, Albuquerque, NM 87131, USA. ${ }^{4}$ Institute of Animal Genetics and Breeding, College of Animal Science and Technology, Sichuan Agricultural University, Chengdu 611130, China. ${ }^{5}$ Department of Chemistry, College of Life and Basic Science, Sichuan Agricultural University, Chengdu 611130, China.

Received: 4 July 2018 Accepted: 6 September 2018 Published online: 20 September 2018

\section{References}

1. Bethony J, Brooker S, Albonico M, Geiger SM, Loukas A, Diemert D, et al. Soil-transmitted helminth infections: ascariasis, trichuriasis, and hookworm. Lancet. 2006;367:1521-32.

2. Bundy DA, Cooper ES. Trichuris and trichuriasis in humans. Adv Parasitol. 1989;28:107-73.

3. Betson M, Søe MJ, Nejsum P. Human trichuriasis: whipworm genetics, phylogeny, transmission and future research directions. Curr Trop Med Rep. 2015;2:209-17.

4. Cleaveland S, Laurenson MK, Taylor LH. Diseases of humans and their domestic mammals: pathogen characteristics, host range and the risk of emergence. Philos Trans R Soc Lond B Biol Sci. 2001;356:991.

5. Anderson RC. Nematode Parasites of Vertebrates: Their Development and Transmission. 2nd ed. Wallingford: CAB International; 2000.

6. Hotez PJ, Molyneux DH, Fenwick A, Kumaresan J, Sachs SE, Sachs JD, et al. Control of neglected tropical diseases. N Engl J Med. 2007;357:1018-27.

7. Schmidt GD, Roberts LS. Orders Trichurata and Dioctophymata: Aphasmidian parasites. In: Schmidt GD, Roberst LS, editors. Foundations of Parasitology. 3rd ed. St. Louis: Times mirror/Mosby; 1985. p. 450-2.

8. Callejón R, Robles MDR, Panei CJ, Cutillas C. Molecular diversification of Trichuris spp. from Sigmodontinae (Cricetidae) rodents from Argentina based on mitochondrial DNA sequences. Parasitol Res. 2016;115:1-13.

9. Taylor LH, Latham SM, Woolhouse MEJ. Risk factors for human disease emergence. Philos Trans R Soc Lond B Biol Sci. 2001;356:983-9.

10. Liu GH, Zhou W, Nisbet AJ, Xu MJ, Zhou DH, Zhao GH, et al. Characterization of Trichuris trichiura from humans and $T$. suis from pigs in
China using internal transcribed spacers of nuclear ribosomal DNA. J Helminthol. 2014:88:64-8.

11. Cutillas C, Callejón R, Rojas MD, Tewes B, Ubeda JM, Ariza C, et al. Trichuris suis and Trichuris trichiura are different nematode species. Acta Trop. 2009; 111:299-307.

12. Nissen S, Aljubury A, Hansen TV, Olsen A, Christensen H, Thamsborg SM, et al Genetic analysis of Trichuris suis and Trichuris trichiura recovered from humans and pigs in a sympatric setting in Uganda. Vet Parasitol. 2012;188:68-77.

13. Liu GH, Gasser RB, Su A, Nejsum P, Peng L, Lin RQ, et al. Clear genetic distinctiveness between human- and pig-derived Trichuris based on analyses of mitochondrial datasets. PLoS Negl Trop Dis. 2012;6:e1539.

14. Munene E, Otsyula M, Mbaabu DA, Mutahi WT, Muriuki SM, Muchemi GM. Helminth and protozoan gastrointestinal tract parasites in captive and wildtrapped African non-human primates. Vet Parasitol. 1998;78:195-201.

15. Dawet A, Yakubu DP, Butu HM. Survey of gastrointestinal parasite of nonhuman primates in Jos Zoological Garden. J Primatol. 2013;2:1.

16. Adetunji VE. Prevalence of gastrointestinal parasites in primates and their keepers from two zoological gardens in Ibadan. Nigeria. Sokoto J Vet Sci. 2014;12:25-30.

17. Mbora DNM, Munene E. Gastrointestinal parasites of critically endangered primates endemic to Tana River, Kenya: Tana River red colobus (Procolobus rufomitratus) and crested mangabey (Cercocebus galeritus). J Parasitol. 2006; 92:928-32.

18. Phillips KA, Haas ME, Grafton BW, Yrivarren M. Survey of the gastrointestinal parasites of the primate community at Tambopata National Reserve, Peru. J Zool. 2010;264:149-51.

19. Levecke B, Dorny P, Vercammen F, Visser LG, Van EM, Vercruysse J, et al. Transmission of Entamoeba nuttalli and Trichuris trichiura from non-human primates to humans. Emerg Infect Dis. 2015;21:1871-2.

20. Melfi V, Poyser F. Trichuris burdens in zoo-housed Colobus guereza. Int J Primatol. 2007;28:1449-56.

21. Son Vo Dinh. Intestinal parasites of Macaca fascicularis in a mangrove forest. Ho Chi Minh City: Laboratory Primate Newsletter; 2002

22. Taylor L, Lessnau RG, Lehman SM. Prevalence of whipworm (Trichuris) ova in two free-ranging populations of rhesus macaques (Macaca mulatta) in the Florida Keys. Florida Scientist. 1994:57:102-7.

23. Ravasi DF, O'Riain MJ, Davids F, Illing N. Phylogenetic evidence that two distinct Trichuris genotypes infect both humans and non-human primates. PLoS One. 2012;7:e44187.

24. Callejón R, Halajian A, Cutillas C. Description of a new species, Trichuris ursinus n. sp.(Nematoda: Trichuridae) from Papio ursinus Keer, 1792 from South Africa. Infect Genet Evol. 2017:51:182-93.

25. Hansen TV, Thamsborg SM, Olsen A, Prichard RK, Nejsum P. Genetic variations in the beta-tubulin gene and the internal transcribed spacer 2 region of Trichuris species from man and baboons. Parasit Vectors. 2013;6:236.

26. Ghai RR, Simons ND, Chapman CA, Omeja PA, Davies TJ, Ting N, et al. Hidden population structure and cross-species transmission of whipworms (Trichuris sp.) in humans and non-human primates in Uganda. PLoS Negl Trop Dis. 2014:8:e3256

27. Liu GH, Gasser RB, Nejsum P, Wang Y, Chen Q, Song HQ, et al. Mitochondrial and nuclear ribosomal DNA evidence supports the existence of a new Trichuris species in the endangered François' leaf-monkey. PLoS One. 2013:8:e66249.

28. Hawash MB, Andersen LO, Gasser RB, Stensvold CR, Nejsum P. Mitochondria genome analyses suggest multiple Trichuris species in humans, baboons, and pigs from different geographical regions. PLoS Negl Trop Dis. 2015;9: e0004059.

29. Elliott DE, Summers RW, Weinstock JV. Helminths as governors of immunemediated inflammation. Int J Parasitol. 2007;37:457-64.

30. Jenkins T. A morphological and histochemical study of Trichuris suis (Schrank, 1788) with special reference to the host-parasite relationship. Parasitology. 1970;61:357-74.

31. Ooi HK, Tenora F, Itoh K, Kamiya M. Comparative study of Trichuris trichiura from non-human primates and from man, and their difference with $T$. suis. J Vet Med Sci. 1993;55:363-6.

32. Zhou X, Yu H, Wang N, Xie Y, Liang YN, Li DS, et al. Molecular diagnosis of Baylisascaris schroederi infections in giant panda (Ailuropoda melanoleuca) faeces using PCR. J Wildl Dis. 2013:49:1052-5.

33. Thompson JD, Gibson TJ, Plewniak F, Jeanmougin F, Higgins DG. The CLUSTAL X windows interface: flexible strategies for multiple sequence alignment aided by quality analysis tools. Nucleic Acids Res. 1997;25:4876-82. 
34. Tamura K, Stecher G, Peterson D, Filipski A, Kumar S. MEGA6: Molecular Evolutionary Genetics Analysis version 6.0. Mol Biol Evol. 2013;30:2725-9.

35. Swofford DL. PAUP*. Phylogenetic analysis using parsimony (and other methods). Sunderland: Sinauer Associates; 2002.

36. Ronquist F, Teslenko M, van der Mark P, Ayres DL, Darling A, Höhna S, et al. MrBayes 3.2: efficient Bayesian phylogenetic inference and model choice across a large model space. Syst Bio. 2012;61:539-42.

37. Xie Y, Hoberg EP, Yang Z, Urban JF, Yang G. Ancylostoma ailuropodae n. sp. (Nematoda: Ancylostomatidae), a new hookworm parasite isolated from wild giant pandas in southwest China. Parasit Vectors. 2017;10:277.

38. Darriba D, Taboada GL, Doallo R, Posada D. jModelTest 2: more models, new heuristics and parallel computing. Nat Methods. 2012;9:772.

39. Page RD. Tree View: an application to display phylogenetic trees on personal computers. Comput Appl Biosci. 1996;12:357-8.

40. Lavrov DV, Brown WM. Trichinella spiralis mtDNA: a nematode mitochondrial genome that encodes a putative ATP8 and normally structured tRNAS and has a gene arrangement relatable to those of coelomate metazoans. Genetics. 2001;157:621-37.

41. Mohandas N, Pozio E, La Rosa G, Korhonen PK, Young ND, Koehler AV, et al. Mitochondrial genomes of Trichinella species and genotypes - a basis for diagnosis, and systematic and epidemiological explorations. Int J Parasitol. 2014:44:1073-80.

42. Cavallero S, De Liberato C, Friedrich KG, Di Cave D, Masella V, D'Amelio S, et al. Genetic heterogeneity and phylogeny of Trichuris spp. from captive nonhuman primates based on ribosomal DNA sequence data. Infect Genet Evol. 2015;34:450-6.

43. Hawash MB, Betson M, Al-Jubury A, Ketzis J, Leewillingham A, Bertelsen MF, et al. Whipworms in humans and pigs: origins and demography. Parasit Vectors. 2016;9:37.

44. Leles D, Gardner SL, Reinhard K, Iñiguez A, Araujo A. Are Ascaris /umbricoides and Ascaris suum a single species? Parasit Vectors. 2012;5:42.

45. Liu GH, Wu CY, Song HQ, Wei SJ, Xu MJ, Lin RQ, et al. Comparative analyses of the complete mitochondrial genomes of Ascaris lumbricoides and Ascaris suum from humans and pigs. Gene. 2012:492:110-6.

46. Mattiucci S, Cipriani P, Webb SC, Paoletti M, Marcer F, Bellisario B, et al. Genetic and morphological approaches distinguish the three sibling species of the Anisakis simplex species complex, with a species designation as Anisakis berlandi n. sp. for A. simplex sp. C (Nematoda: Anisakidae). J Parasitol. 2014;100:199-214.

47. Zhu XQ, Jacobs DE, Chilton NB, Sani RA, Cheng NA, Gasser RB. Molecular characterization of a Toxocara variant from cats in Kuala Lumpur, Malaysia. Parasitology. 1998;117:155-64.

48. Hu M, Chilton NB, Gasser RB. The mitochondrial genomics of parasitic nematodes of socio-economic importance: recent progress, and implications for population genetics and systematics. Adv Parasitol. 2003;56: $133-212$.

49. Zhan B, Li T, Xiao S, Zheng F, Hawdon J. Species-specific identification of human hookworms by PCR of the mitochondrial cytochrome oxidase I gene. J Parasitol. 2001:87:1227-9.

50. Blouin MS, Yowell CA, Courtney CH, Dame JB. Substitution bias, rapid saturation, and the use of mtDNA for nematode systematics. Mol Biol Evol. 1998;15:1719-27.

51. Zhu C, Tong X, Wu D. A new species of Trichocephalus (Trichocephalidae) from the golden monkey (Rhinopipthecus roxellanae). J Sichuan Inst Anim Husb Vet Med. 2000;14:25-31.

52. Hou Y, Peng Y. A description of new species of Trichocephalus (Trichocephalidae, Trichocephalata) from the golden monkey, Rhinopithecus bieti. Zool Res. 1989;10:45-50.

53. Tandonnet S, Pires-Dasilva A. Phenotypic plasticity and developmental innovations in nematodes. Curr Opin Genet Dev. 2016;39:8-13.

54. Viney ME. Life-history plasticity and responses to host defense. In: Kennedy MW, Harnett W, editors. Parasitic Nematodes: Molecular Biology, Biochemistry and Immunology. Wallingford: CAB International; 2013. p. 15-29.

55. Bitnermathé BC, Klaczko LB. Plasticity of Drosophila melanogaster wing morphology: effects of sex, temperature and density. Genetica. 1999;105: 203-10

56. Castaneda LE, Sabat P, Gonzalez SP, Nespolo RF. Digestive plasticity in tadpoles of the Chilean giant frog (Caudiverbera caudiverbera): factorial effects of diet and temperature. Physiol Biochem Zool. 2006;79:919-26.

57. Kiontke K, Fitch DH. Phenotypic plasticity: different teeth for different feasts. Curr Biol. 2010;20:R710-2.
58. Viney ME, Diaz A. Phenotypic plasticity in nematodes: evolutionary and ecological significance. Worm. 2012;1:98-106.

59. Mati VL, Freitas RM, Bicalho RS, Melo AL. Phenotypic plasticity of male Schistosoma mansoni from the peritoneal cavity and hepatic portal system of laboratory mice and hamsters. J Helminthol. 2015;89:294-301.

60. Callejón R, Cutillas C, Nadler SA. Nuclear and mitochondrial genes for inferring Trichuris phylogeny. Parasitol Res. 2015;114:4591-9.

61. Thomas WK, Wilson AC. Mode and tempo of molecular evolution in the nematode Caenorhabditis: cytochrome oxidase II and calmodulin sequences. Genetics. 1991;128:269-79.

62. Xie Y, Zhang Z, Wang C, Lan J, Li Y, Chen Z, et al. Complete mitochondrial genomes of Baylisascaris schroederi, Baylisascaris ailuri and Baylisascaris transfuga from giant panda, red panda and polar bear. Gene. 2011:482:59-67.

63. Agosta SJ, Janz N, Brooks DR. How specialists can be generalists: resolving the "parasite paradox" and implications for emerging infectious disease. Zoologia. 2010;27:151-62.

64. Araujo SB, Braga MP, Brooks DR, Agosta SJ, Hoberg EP, von Hartenthal FW, et al. Understanding host-switching by ecological fitting. PLoS One. 2015; e0139225:10.

65. Hoberg EP, Brooks DR. Evolution in action: climate change, biodiversity dynamics and emerging infectious disease. Philos Trans R Soc Lond B Biol Sci. 2015:370:20130553.

66. Nylin S, Agosta S, Bensch S, Boeger WA, Braga MP, Brooks DR, et al. Embracing colonizations: a new paradigm for species association dynamics. Trends Ecol Evol. 2018;33:4-14.

67. Hoberg EP, Brooks DR. A macroevolutionary mosaic: episodic hostswitching, geographic colonization, and diversification in complex hostparasite systems. J Biogeogr. 2008;35:1533-50.

\section{Ready to submit your research? Choose BMC and benefit from:}

- fast, convenient online submission

- thorough peer review by experienced researchers in your field

- rapid publication on acceptance

- support for research data, including large and complex data types

- gold Open Access which fosters wider collaboration and increased citations

- maximum visibility for your research: over $100 \mathrm{M}$ website views per year

At $\mathrm{BMC}$, research is always in progress.

Learn more biomedcentral.com/submissions 